\title{
3D Stochastic Completion Fields for Fiber Tractography
}

\author{
Parya Momayyez \\ School of Computer Science \\ Centre for Intelligent Machines \\ McGill University, Montréal, QC, Canada \\ pmamay@cim.mcgill.ca
}

\author{
Kaleem Siddiqi \\ School of Computer Science \\ Centre for Intelligent Machines \\ McGill University, Montréal, QC, Canada \\ siddiqiecim.mcgill.ca
}

\begin{abstract}
We approach the problem of fiber tractography from the viewpoint that a computational theory should relate to the underlying quantity that is being measured - the diffusion of water molecules. We characterize the brownian motion of water by a $3 D$ random walk described by a stochastic nonlinear differential equation. We show that the maximumlikelihood trajectories are $3 D$ elastica, or curves of least energy. We illustrate the model with Monte-Carlo (sequential) simulations and then develop a more efficient (local, parallelizable) implementation, based on the Fokker-Planck equation. The final algorithm allows us to efficiently compute stochastic completion fields to connect a source region to a sink region, while taking into account the underlying diffusion MRI data. We demonstrate promising tractography results using high angular resolution diffusion data as input.
\end{abstract}

\section{Introduction}

Diffusion magnetic resonance imaging (DMRI) [11] provides a noninvasive means to explore neural connectivity between distinct areas of the brain, a subject of significant interest in neuroscience [21]. DMRI is based on the in-vivo measurement of the brownian motion of water molecules in living organisms, and on the property that it is anisotropic in brain white matter and other fibrous tissues. This anisotropy is primarily caused by the presence of bundles of axons, which restrict the diffusion of water in the direction perpendicular to them. The direction of maximal diffusion is therefore assumed to coincide with the direction of fiber tracts [12].

There is a growing body of research in various aspects of DMRI including low-level tasks such as data acquisition and modeling, regularization, sharpening and the extraction of invariant measures, as well as higher-level ones such as fiber tractography. These tasks are all complicated by the fact that the resolution of DMRI is typically on the order of $8 \mathrm{~mm}^{3}$ while the diameter of the individual fibers is between 1-30 $\mu \mathrm{m}$. As a result, partial volume averaging effects occur when more than one family of fibers are in a crossing, branching, or merging configuration within the same voxel.

This article focuses on the higher-level task of fiber tractography, where the goal is to begin with DMRI data as input and then find putative fiber tracts between distinct regions. Measures of connectivity between the regions are then typically obtained as a post-processing step. We depart from most traditional tractography approaches in two ways. First, we develop a computational model that is fundamentally linked to the diffusion process itself, i.e., it characterizes the diffusion of water molecules in a medium. Second, we estimate putative tracts between a source and a sink region, along with their probabilities of occurring, simultaneously. We accomplish this by extending Williams and Jacobs' curve completion model in computer vision [24] to 3D. Their model has been applied to contour completion tasks in computer vision, but to our knowledge it has not yet been formulated in 3D nor has it been used for DMRI analysis.

Our main contributions are: 1) the introduction of the 3D stochastic completion field to characterize the brownian motion of water molecules in 3D and 2) an efficient (local and parallelizable) implementation of the model based on the Fokker-Planck equation. We utilize the method to obtain probabilistic estimates of fiber tracts between two seed regions selected from the corpus callosum, the cingulum and the cortico-spinal tract. We begin with a brief review of tractography methods in DMRI.

\section{Background}

\subsection{Deterministic Tractography}

Deterministic tractography methods seek to find the most likely paths of connection between different regions, but without an explicit probability model to capture the effects 
of signal noise, partial volume averaging or other artifacts. The confidence in a path or a connectivity measure is usually obtained by examining the degree to which a recovered path satisfies specified constraints, or by counting the number of times the generated tracts pass through a specific voxel $[16,17,5]$. These techniques can be classified into two major groups: streamline tracking and energy minimization-based tracking.

Streamline tracking techniques $[13,1,7]$ propagate the fiber path in a sequential manner using the local diffusion tensor (DT) or orientation distribution function (ODF). Mori et al.'s fiber assignment by continuous tracking (FACT) algorithm starts from a seed point and follows the direction of the tensor's major eigenvector [13]. To avoid generating discretized trajectories, a continuous smooth trajectory is followed. Basser et al. [1] have used an interpolation scheme to approximate a continuous tensor field in order to evolve fiber tracts described by a differential equation. Although the generalization of streamline tracking to use HARDI data can lead to improvements [19], the possibility of swerving away from a true trajectory, due to noise and imaging artifacts, still exists.

Energy minimization-based tracking algorithms [16, 17, $6,5]$ seek to find the curves which fit the ODF/DT maxima while minimizing a predefined energy or cost function. The methods of $[16,5]$ use an eikonal equation to evolve a surface away from a seed region, using a level-set embedding, with a speed function that is proportional to the likelihood of diffusion in a particular direction. The recovered tracts are obtained by using gradient descent on a time of arrival map. A different class of methods combines a priori knowledge of the most probable fascicle directions with the diffusion data. For example, using a framework of spin glasses, where one or more spins are placed in each voxel [6], a global regularization scheme can be carried out which associates low curvature or low bending energy constraints with fiber trajectories. This framework can compensate for partial volume effects by choosing a more plausible orientation when the major eigenvector is far from the true fiber orientation, and the presence of two or more spins in each voxel can be used to signal fiber crossings [6].

Although energy/cost minimization techniques can be used to define a connectivity measure in which the uncertainty of DMRI data is embedded, the explicit use of a probability model to capture the effects of noise and other imaging artifacts (independent of the tractography method being used) and its incorporation into each tractography step is a more principled alternative. Probabilistic tractography methods deal with this challenge to some extent.

\subsection{Probabilistic Tractography}

Probabilistic tractography methods incorporate the local uncertainty resulting from different artifacts at each voxel during fiber propagation. These techniques can be divided into two main groups: those which describe the local uncertainty by some probability distribution function (PDF) $[15,3,2]$, and bootstrap based methods which capture the uncertainty of the data by random selection from a set of different measurements [8, 10, 4].

Among the first class of methods, Behrens et al. [3] estimate the local uncertainty associated with a diffusion model at each voxel by computing the posterior distribution on the parameters of the corresponding model, given the diffusion data. Associating a prior probability with the model parameters, a Markov Chain Monte Carlo (MCMC) sampling scheme is implemented for the numerical estimation of the local PDFs. The local uncertainty of model parameters is later used in a streamline tractography framework to compute inter-region connectivity measures. This framework has been extended in [2] by acquiring more diffusion images which are used for Bayesian estimation of the parameters of a multi-fiber diffusion model to handle complex fiber architectures.

Whereas the above approaches show an improvement over the deterministic ones, due to their dependence on the underlying noise model and the prior distributions, the estimated PDFs may not always provide an accurate representation of the true noise and imaging artifacts. Bootstrap based probabilistic approaches [8, 10, 4] have circumvented this limitation to an extent by a non-parametric estimation of diffusion uncertainty. Jones et Pierpaoli's [8] bootstrap based method draws random samples from a limited number of DTI acquisitions to construct a large set of bootstrapped DTI volumes on which streamline tractography is carried out. A similar approach has been taken by Lazar et al. [10], where an estimate of tract dispersion is obtained at each voxel from the $2 \mathrm{D}$ distribution of fiber orientations in the plane normal to the fiber direction.

\section{3D Stochastic Completion Fields}

We depart from earlier tractography methods by considering the physical process which underlies DMRI. We model the diffusion of water molecules in a medium by a random walk, which is an extension of Williams and Jacobs' curve completion model in computer vision [24]. A particle's state in $\mathbb{R}^{3} \times S^{2}$ (its position and orientation) is updated using the following non-linear stochastic differential equation:

$$
\begin{aligned}
& \dot{x}=\sin \theta \cdot \cos \phi ; \quad \dot{y}=\sin \theta \cdot \sin \phi ; \quad \dot{z}=\cos \theta \\
& \dot{\theta}=N\left(0, \sigma_{\theta}^{2}\right) ; \quad \dot{\phi}=N\left(0, \sigma_{\phi}^{2}\right) .
\end{aligned}
$$

Following [24], we also allow a certain fraction of particles $\left(1-e^{-\frac{1}{\zeta}}\right)$ to decay per unit time to give greater support to shorter paths. The key intuition is that a particle should 


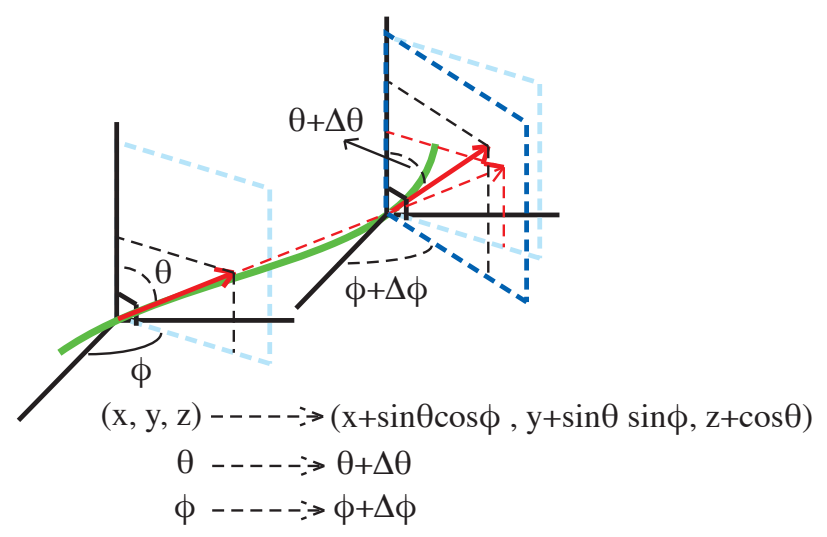

Figure 1. The 3D random walk. A particle takes a small step in the direction it is presently heading, and then the tangent vector is rotated by $\Delta \theta$ and $\Delta \phi$ in the osculating and binormal planes, respectively.
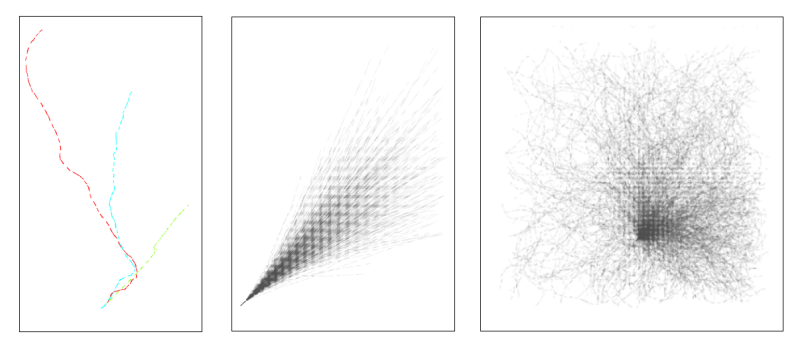

Figure 2. A number of trajectories obtained using the 3D random walk model with Monte-Carlo simulation. LEFT: Three trajectories with different values of $\sigma_{\theta}$ and $\sigma_{\phi}$. MIDDLE: A stochastic source field with $\sigma_{\phi}=\sigma_{\theta}=0.02, \zeta=150$. RIGHT: A stochastic source field with $\sigma_{\phi}=\sigma_{\theta}=0.2, \zeta=150$. In the middle and right panels transparency is inversely proportional to the probability of reaching a particular state.

move with constant speed in a direction which is continually changing (slightly). These changes are described by two successive deviations (see Fig. 1.) The first is a deviation by an amount proportional to $\sigma_{\phi}^{2}$ in the $x y$-plane. This corresponds to the amount of rotation in the osculating plane, i.e., the plane containing the tangent and normal vectors of the local Frenet Frame. The second is a deviation by an amount proportional to $\sigma_{\theta}^{2}$ in the plane which contains the current tangent vector and is perpendicular to the $x y$-plane. This corresponds to the amount of rotation in the binormal plane, i.e., the plane containing the tangent and binormal vectors of the local Frenet Frame.

The main motivation for the above formulation is that it allows us to use all machinery developed by Williams and Jacobs for the 2D case (see [24]) to calculate the probability $p(x, y, z, \theta, \phi ; t)$ that a particle beginning in a particular source state $\left(x_{0}, y_{0}, z_{0}, \theta_{0}, \phi_{0}\right)$ will reach an arbitrary state $(x, y, z, \theta, \phi)$ in time $t$ before decaying. In fact, the nec- essary integral can be computed by convolution, exploiting the translational and rotation symmetries of the Green's function $G$. In the 3D setting, $G$ is a rank ten tensor representing the transition probabilities of a Markov process defined on the five-dimensional state space $\mathbb{R}^{3} \times S^{2}$, while satisfying Eq. 1. This leads to the notion of a stochastic source field $p(x, y, z, \theta, \phi)$ which represents the fraction of paths which begin in a source state $\left(x_{0}, y_{0}, z_{0}, \theta_{0}, \phi_{0}\right)$ and pass through state $(x, y, z, \theta, \phi)$ before decaying. Fig. 2 illustrates a few sample trajectories generated by the random walk (left) and then two different stochastic source fields obtained with different parameters but beginning from the same initial state (middle and right). As the reader will note, with small values of $\sigma_{\theta}, \sigma_{\phi}$ the stochastic source field has a dominant direction, while as these values grow large the field resembles true brownian motion of water in an isotropic medium.

A very similar construction leads to the notion of a stochastic sink field, which represents the probability that a particle beginning in a state $(x, y, z, \theta, \phi)$ will reach a sink state before decaying. The stochastic completion field is then the product of these two fields [24]. It represents the probabilities of paths beginning in a source state and ending in a sink state. In other words, it provides a true probabilistic view of likely trajectories between a source and sink state. We shall show that when combined with DMRI data, the stochastic completion field provides a suitable basis for probabilistic fiber tractography.

\section{Relationship to Elastica or Curves of Least Energy}

Using the Markovian property, the paths generated by the random walk can be obtained by a number of independent identically distributed (i.i.d.) discrete steps. It can be shown that in analogy to the $2 \mathrm{D}$ case, the maximum likelihood (discrete) paths correspond to a form of 3D elastica or curves of least energy. Imagine having a source voxel at $p$ and a sink voxel at $q$. A given particle begins its random walk from $p$ following a path called $\Gamma_{p}$ which consists of $n$ unit length steps. At each step there are two changes in the orientation, change in $\phi$ and change in $\theta$, given by $\kappa_{1}, \ldots, \kappa_{n}$ and $\tau_{1}, \ldots, \tau_{n}$. Adopting the notation in [24], the density function for the set of paths leaving source voxel $p$ (under the i.i.d. assumption) is given by:

$$
f\left(\Gamma_{p}\right)=\prod_{i=1}^{n} e^{-\frac{1}{\zeta}} \frac{1}{\sigma_{\phi} \sqrt{2 \pi}} e^{-\frac{\kappa_{i}^{2}}{2 \sigma_{\phi}^{2}}} \frac{1}{\sigma_{\theta} \sqrt{2 \pi}} e^{-\frac{\tau_{i}^{2}}{2 \sigma_{\theta}^{2}}} .
$$

Next, to get the density function for the set of paths starting at source $p$ and ending in sink $q$, i.e. $\Gamma_{p q}$, the above density function is divided by the integration of $f\left(\Gamma_{p}\right)$ over all 
paths ending in $q$, i.e. $\Gamma_{q}$.

$$
\begin{aligned}
g\left(\Gamma_{p q}\right) & =\frac{1}{\int_{\Gamma_{q}} f\left(\Gamma_{p}\right) d \Gamma_{p}} \\
& \times \prod_{i=1}^{n} e^{-\frac{1}{\zeta}} \frac{1}{\sigma_{\phi} \sqrt{2 \pi}} e^{-\frac{\kappa_{i}^{2}}{2 \sigma_{\phi}^{2}}} \frac{1}{\sigma_{\theta} \sqrt{2 \pi}} e^{-\frac{\tau_{i}^{2}}{2 \sigma_{\theta}^{2}}}
\end{aligned}
$$

Taking logarithms of both sides, we have:

$$
\begin{aligned}
\log g\left(\Gamma_{p q}\right)+C & =\frac{-n}{\zeta} \\
& -n \log \left(\sigma_{\phi} \sqrt{2 \pi}\right)-n \log \left(\sigma_{\theta} \sqrt{2 \pi}\right) \\
& -\sum_{i=1}^{n} \frac{\kappa_{i}^{2}}{2 \sigma_{\phi}^{2}}-\sum_{i=1}^{n} \frac{\tau_{i}^{2}}{2 \sigma_{\theta}^{2}}
\end{aligned}
$$

Now consider continuous curves which minimize the following energy in 3D

$$
\alpha \int_{\Gamma} \kappa(t)^{2} d t+\beta \int_{\Gamma} \tau(t)^{2} d t+\gamma \int_{\Gamma} d t
$$

which is a weighted linear combination of length, curvature squared and torsion squared. Equation (5) provides a natural extension of the notion of elastica in 2D (as in [14]) to 3D. In the discrete case, in Eq. (4), the orientation changes in the osculating plane $\kappa_{1}, \ldots, \kappa_{n}$ correspond to curvature values and those in the binormal plane $\tau_{1}, \ldots, \tau_{n}$ to torsion values. If we consider polygonal arcs with $n$ segments having curvature and torsion values of $\kappa_{i}$ and $\tau_{i}$ respectively, the maximum likelihood paths in Eq. (4) can be seen to minimize a weighted sum of length, curvature squared and torsion squared, with $\alpha=\frac{1}{2 \sigma_{\phi}^{2}}, \beta=\frac{1}{2 \sigma_{\theta}^{2}}$ and $\gamma=\frac{1}{\zeta}+\log \left(\sigma_{\theta} \sqrt{2 \pi}\right)+\log \left(\sigma_{\phi} \sqrt{2 \pi}\right)$.

\section{Differential Equation for the 3D Random Walk}

We now exploit the Fokker-Planck equation [18], which describes the time evolution of the probability density of the particles following a random walk in space, to develop an efficient (local and parallelizable) implementation of the random walk. Our development is inspired by the course notes of Martin Bazant available at "http://math.mit.edu/18.366/lec06/". Using the Markov property, the probability density function $P_{N+1}(X)$ after $N+1$ steps for a given state $X$ can be written as $P_{N+1}(X)=\int p_{N}\left(X \mid X^{\prime}\right) P_{N}\left(X^{\prime}\right) d X^{\prime}$. Defining a function $P(X, t)$ such that $P(X, N \Delta t)=$ $P_{N}(X)$, where $t=N \Delta t$ is a continuous time variable, and a new transition probability function as $p\left(X, t+\Delta t \mid X^{\prime}, t\right)=p_{N}\left(X \mid X^{\prime}\right)$, it follows that $P(X, t+\Delta t)=\int p\left(X, t+\Delta t \mid X^{\prime}, t\right) P\left(X^{\prime}, t\right) d X^{\prime} . \mathrm{A}$ partial differential equation can be derived for the function $P(X, t)$ as $N$ goes to infinity. Next, considering the limit as $\Delta t \rightarrow 0$ leads to the Fokker-Planck equation (with $X=\left\{X_{1}, \ldots, X_{n}\right\}$ an $N$-dimensional variable):

$$
\begin{aligned}
& \frac{\partial P}{\partial \Delta t}+\sum_{i=1}^{N} \frac{\partial}{\partial X_{i}}\left(D_{(i)}^{(1)}(X, t) P\right)=\sum_{i=1}^{N} \frac{\partial^{2}}{\partial X_{i}^{2}}\left(D_{(i)}^{(2)}(X, t) P\right) \\
& D_{(i)}^{(1)}(X, t)=\lim _{\Delta t \rightarrow 0} \frac{M_{(i)}^{(1)}(X, t)}{\Delta t} \\
& D_{(i)}^{(2)}(X, t)=\lim _{\Delta t \rightarrow 0} \frac{M_{(i)}^{(2)}(X, t)}{2 \Delta t} \\
& M_{(i)}^{(n)}(X, t)=\left\langle\left(X_{i}^{\prime}-X_{i}\right)^{n}\right\rangle \\
& =\int p\left(X_{i}^{\prime}, t+\Delta t \mid X_{i}, t\right)\left(X_{i}^{\prime}-X_{i}\right)^{n} d X_{i}^{\prime},
\end{aligned}
$$

Using Equation 1 which describes the random walk implemented for 3D completion field, and defining a given state $X$ as $(x, y, z, \theta, \phi)$, the corresponding Fokker-Planck equation can now be stated:

$$
\begin{aligned}
D_{(x)}^{(1)}(X, t) & =\lim _{\Delta t \rightarrow 0} \frac{\int p\left(x^{\prime}, t+\Delta t \mid x, t\right)\left(x^{\prime}-x\right) d x^{\prime}}{\Delta t} \\
& =\lim _{\Delta t \rightarrow 0} \frac{\int \delta\left(x^{\prime}-x-\Delta t \sin \theta \cos \phi\right)\left(x^{\prime}-x\right) d x^{\prime}}{\Delta t} \\
& =\lim _{\Delta t \rightarrow 0} \frac{\Delta t \sin \theta \cos \phi}{\Delta t}=\sin \theta \cos \phi \\
D_{(x)}^{(2)}(X, t) & =\lim _{\Delta t \rightarrow 0} \frac{\int p\left(x^{\prime}, t+\Delta t \mid x, t\right)\left(x^{\prime}-x\right)^{2} d x^{\prime}}{2 \Delta t} \\
& =\lim _{\Delta t \rightarrow 0} \frac{\int\left(x^{\prime}-x-\Delta t \sin \theta \cos \phi\right)\left(x^{\prime}-x\right)^{2} d x^{\prime}}{2 \Delta t} \\
& =\lim _{\Delta t \rightarrow 0} \frac{(\Delta t)^{2} \sin ^{2} \theta \cos ^{2} \phi}{\Delta t}=0 .
\end{aligned}
$$

$D_{(y)}^{(1)}(X, t), D_{(y)}^{(2)}(X, t), D_{(z)}^{(1)}(X, t)$ and $D_{(z)}^{(2)}(X, t)$ are derived in a similar manner, resulting in:

$$
\begin{array}{r}
D_{(y)}^{(1)}(X, t)=\sin \theta \sin \phi, \\
D_{(z)}^{(1)}(X, t)=\cos \theta, \\
D_{(y)}^{(2)}(X, t)=D_{(z)}^{(2)}(X, t)=0 .
\end{array}
$$

Next, we need to derive the corresponding $D$ values for $\theta$ and $\phi$. It is worth mentioning that, since the changes in $\theta$ and $\phi$ after each unit step are normally distributed with sigma values of $\sigma_{\theta}$ and $\sigma_{\phi}$, the corresponding changes in $\theta$ and $\phi$ after a step equal to $\Delta t$ are governed by normal distributions, with modified sigma values of $\sigma_{\theta} \sqrt{\Delta t}$ and $\sigma_{\phi} \sqrt{\Delta t}$. 
Using this, we will have:

$$
\begin{aligned}
D_{(\theta)}^{(1)}(X, t) & =\lim _{\Delta t \rightarrow 0} \frac{\int p\left(\theta^{\prime}, t+\Delta t \mid \theta, t\right)\left(\theta^{\prime}-\theta\right) d \theta^{\prime}}{\Delta t} \\
& =\lim _{\Delta t \rightarrow 0} \frac{\int \frac{1}{\sqrt{2 \pi \Delta t \sigma_{\theta}^{2}}} \exp \left(\frac{-\Delta \theta^{\prime 2}}{2 \Delta t \sigma_{\theta}^{2}}\right) \Delta \theta^{\prime} d \Delta \theta^{\prime}}{\Delta t} \\
& =\lim _{\Delta t \rightarrow 0} \frac{\text { Mean value of the normal distribution }}{\Delta t} \\
& =0 \\
D_{(\theta)}^{(2)}(X, t) & =\lim _{\Delta t \rightarrow 0} \frac{\int p\left(\theta^{\prime}, t+\Delta t \mid \theta, t\right)\left(\theta^{\prime}-\theta\right)^{2} d \theta^{\prime}}{\Delta t} \\
& =\lim _{\Delta t \rightarrow 0} \frac{\int \frac{1}{\sqrt{2 \pi \Delta t \sigma_{\theta}^{2}}} \exp \left(\frac{-\Delta \theta^{\prime 2}}{2 \Delta t \sigma_{\theta}^{2}}\right) \Delta^{2} \theta^{\prime} d \Delta \theta^{\prime}}{2 \Delta t} \\
& =\lim _{\Delta t \rightarrow 0} \frac{\text { Variance of the normal distribution }}{2 \Delta t} \\
& =\frac{\sigma_{\theta}^{2} \Delta t}{2 \Delta t}=\frac{\sigma_{\theta}^{2}}{2} .
\end{aligned}
$$

A similar result is obtained for $D_{(\phi)}^{(1)}(X, t)$ and $D_{(\phi)}^{(2)}(X, t)$ :

$$
D_{(\phi)}^{(1)}(X, t)=0, \quad D_{(\phi)}^{(2)}(X, t)=\frac{\sigma_{\phi}^{2}}{2} .
$$

Replacing $\Delta t$ by $t$ for the sake of simplicity, the final Fokker-Planck equation can be written as:

$$
\begin{aligned}
& P\left(x, y, z, \theta, \phi ; t^{\prime}\right)=P(x, y, z, \theta, \phi ; 0) \\
&+ \int_{0}^{t^{\prime}} \frac{\partial P(x, y, z, \theta, \phi ; t)}{\partial t} d t \\
& \frac{\partial P}{\partial t}=-\sin \theta \cos \phi \frac{\partial P}{\partial x}-\sin \theta \sin \phi \frac{\partial P}{\partial y}-\cos \theta \frac{\partial P}{\partial z} \\
&+\frac{\sigma_{\phi}^{2}}{2} \frac{\partial^{2} P}{\partial \phi^{2}}+\frac{\sigma_{\theta}^{2}}{2} \frac{\partial^{2} P}{\partial \theta^{2}}-\frac{1}{\zeta} P .
\end{aligned}
$$

\section{Bayesian Stochastic Completion Field Trac- tography}

In the context of fiber tractography we are interested in those curves of least energy between a source and a sink region that are also supported by the underlying DMRI data. Using Bayes' rule we can treat the stochastic completion field at a particular voxel as a prior probability which needs to be multiplied by a suitable conditional probability to give the posterior probability of that completion field occurring. If we further assume that the presence of a particular ODF (ideally obtained from a high angular resolution diffusion (HARD) dataset such as Q-ball imaging (QBI) [21] or a fiber ODF [20]) is independent of the stochastic completion field, which is a reasonable assumption, we can simply use the underlying DMRI data as the conditional. This leads to two computational methods for stochastic completion field tractography.

\subsection{A Sequential, Global Method}

Given two sets of source and sink voxels, a sequential, global approach can be divided into two steps: (1) Running a Monte-Carlo based 3D-completion field algorithm, for a set of uniformly sampled binormal vectors around each direction in each source and sink voxel and (2) Multiplying the value of the obtained stochastic completion field at each position and orientation by the corresponding QBI or Fiber ODF profile at that location. The second part can be done either by just considering the values inside some cone of uncertainties around ODF maxima or by considering the whole ODF regardless of the orientation of ODF maxima. The method is very easy to implement, but has the disadvantage that its computational complexity is quadratic in the number of source and sink voxels.

\subsection{A Local, Parallelizable Method}

A much more efficient discrete method is based on the Fokker-Planck differential equation developed in Section 5. The Taylor series expansion of the PDE is used to define the probabilities associated with each voxel at time $t+1$ as a function of the values of the nearby voxels at time $t$. Similar to the solution given in [24], the iterative method described in Fig. 3 can give an approximate solution of the Fokker-Planck equation. Here $\lambda_{\theta}=\sigma_{\theta}^{2} / 2(\Delta \theta)^{2}$ and $\lambda_{\phi}=\sigma_{\phi}^{2} / 2(\Delta \phi)^{2}$. Running the above iterative method, the source field, $P^{\prime}(x, y, z, \theta, \phi)$, is computed by integrating $P^{t}(x, y, z, \theta, \phi)$ over time:

$$
P^{\prime}(x, y, z, \theta, \phi)=\int_{0}^{\infty} d t P^{t}(x, y, z, \theta, \phi) .
$$

This integral can be approximated by considering the sequences up to some fixed time $t^{\prime}$ :

$$
P^{\prime}\left(x, y, z, \theta, \phi ; t^{\prime}\right) \approx \sum_{0}^{t^{\prime}} P^{t}(x, y, z, \theta, \phi) .
$$

The sink field can be computed in a similar way, following which one again post-multiplies the obtained stochastic completion field with QBI or fiber ODF data. This method is both local and parallelizable and it has the significant computational advantage that its complexity is linear in the number of source and sink voxels. It also affords the possibility of incorporating local DMRI data in the choice of the parameters $\sigma_{\theta}, \sigma_{\phi}$ of the completion field locally.

\section{Experimental Results}

To validate the performance of the above tractography algorithms, a series of experiments were performed on QBI 


$$
\begin{aligned}
& P_{x, y, z, \theta, \phi}^{t+\frac{1}{6}}=P_{x, y, z, \theta, \phi}^{t}-\sin \theta \cos \phi \begin{cases}P_{x, y, z, \theta, \phi}^{t}-P_{x-1, y, z, \theta, \phi}^{t} & \text { if } \sin \theta \cos \phi>0 \\
P_{x+1, y, z, \theta, \phi}^{t}-P_{x, y, z, \theta, \phi}^{t} & \text { if } \sin \theta \cos \phi<0\end{cases} \\
& P_{x, y, z, \theta, \phi}^{t+\frac{1}{3}}=P_{x, y, z, \theta, \phi}^{t+\frac{1}{6}}-\sin \theta \sin \phi \begin{cases}P_{x, y, z, \theta, \phi}^{t+\frac{1}{6}}-P_{x, y-1, z, \theta, \phi}^{t+\frac{1}{6}} \text { if } \sin \theta \sin \phi>0 \\
P_{x, y+1, z, \theta, \phi}^{t+\frac{1}{6}}-P_{x, y, z, \theta, \phi}^{t+\frac{1}{6}} \text { if } \sin \theta \sin \phi<0\end{cases} \\
& P_{x, y, z, \theta, \phi}^{t+\frac{1}{2}}=P_{x, y, z, \theta, \phi}^{t+\frac{1}{3}}-\cos \theta\left\{\begin{array}{l}
P_{x, y, z, \theta, \phi}^{t+\frac{1}{3}}-P_{x, y, z-1, \theta, \phi}^{t+\frac{1}{3}} \text { if } \cos \theta>0 \\
P_{x, y, z+1, \theta, \phi}^{t+\frac{1}{3}}-P_{x, y, z, \theta, \phi}^{t+\frac{1}{3}} \text { if } \cos \theta<0
\end{array}\right. \\
& P_{x, y, z, \theta, \phi}^{t+\frac{2}{3}}=\lambda_{\theta} P_{x, y, z, \theta-\Delta \theta, \phi}^{t+\frac{1}{2}}+\left(1-2 \lambda_{\theta}\right) P_{x, y, z, \theta, \phi}^{t+\frac{1}{2}}+\lambda_{\theta} P_{x, y, z, \theta+\Delta \theta, \phi}^{t+\frac{1}{2}} \\
& P_{x, y, z, \theta, \phi}^{t+\frac{5}{6}}=\lambda_{\phi} P_{x, y, z, \theta, \phi-\Delta \phi}^{t+\frac{2}{3}}+\left(1-2 \lambda_{\phi}\right) P_{x, y, z, \theta, \phi}^{t+\frac{2}{3}}+\lambda_{\phi} P_{x, y, z, \theta, \phi+\Delta \phi}^{t+\frac{2}{3}} \\
& P_{x, y, z, \theta, \phi}^{t+1}=e^{-\frac{1}{\zeta} P_{x, y, z, \theta, \phi}^{t+\frac{5}{6}}}
\end{aligned}
$$

Figure 3. A local, parallelizable numerical method to solve the Fokker-Planck equation for the 3D stochastic completion field, based on an extension of the technique in [23].

reconstructions of HARD data of the human brain. The diffusion weighted images were acquired in vivo from a healthy subject on a Siemens 3T Trio MR scanner (Siemens Medical Systems, Erlangen, Germany) using an 8-channel phased-array head coil. Diffusion encoding was achieved using a single-shot spin-echo echo planar sequence with twice-refocused balanced diffusion encoding gradients. A dataset designed for high angular resolution reconstruction was acquired with 99 diffusion encoding directions, $2 \mathrm{~mm}$ isotropic voxel size, 63 slices, $b=3000 \mathrm{~s} / \mathrm{mm} 2, T E=121 \mathrm{~ms}$, $\mathrm{TR}=11.1 \mathrm{~s}$, and GRAPPA parallel reconstruction. A $1 \mathrm{~mm}$ isotropic resolution $\mathrm{T} 1$ weighted anatomical scan was also acquired $\left(\mathrm{TR}=9.7 \mathrm{~ms}, \mathrm{TE}=4 \mathrm{~ms}, \alpha=12^{\circ}\right)$. The $\mathrm{QBI}$ reconstruction was based on the technique of Tuch [21].

The performance of both the global and local 3D completion field algorithms was assessed by considering different pairs of ROIs connected through some of the major fiber tracts in the brain. Figure 4 shows the three sets of source and sink regions, marked by white squares on the RGB image of the data. These regions were selected on horizontal, sagittal, and coronal slices respectively to allow for the extraction of part of the corpus callosum, cingulum and cortico-spinal tracts. Each ROI is a $3 \times 3 \times 1$ volume. By setting $\Delta \theta$ and $\Delta \phi$ to $\pi / 10,100$ directions were chosen on one hemisphere which together with their antipodal counterparts was summed to give 200 different directions. $\sigma_{\theta}$ and $\sigma_{\phi}$ were set to 0.2 for all the experiments. The tangent orientations for the source and sink voxels were initialized by finding the ODF maxima in each voxel. Since the direction of water diffusion is not encoded in the diffusion information, both directions corresponding to a given orientation were considered to generate the corresponding source and sink fields. The sequential algorithm was run by uniform sampling of the binormal vector around the initial tangent orientation using a sampling interval of $2 \pi / 36$, and
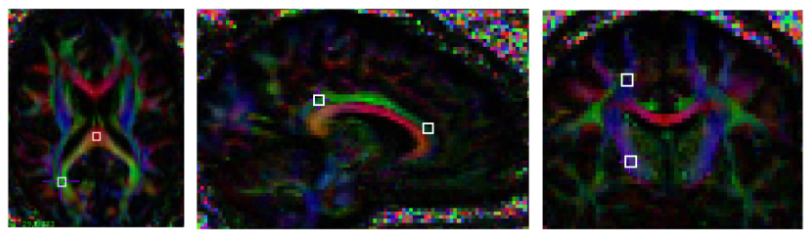

Figure 4. Different source and sink regions are indicated with white squares overlaid on the principal diffusion tensor's direction RGB map. In the RGB images, red represents left-right orientation, green represents anterior-posterior orientation and blue represents inferior-superior orientation. The tract systems are the corpus callosum (left), the cingulum (middle) and the the corticospinal tract (right).

then running 500 trails of the Monte-Carlo simulation for each binormal vector. For the local method, the total number of iterations was set to be the size of the diagonal of the overall volume. This number of iterations is essentially enough for the local algorithm to propagate information to far away points.

Figures 5, 6 and $7{ }^{1}$ show the connectivity paths obtained for the given source and sink regions. The sink and source regions are delimited with green and red blobs. To facilitate the visualization of the recovered paths, the probability of reaching a particular state is inversely proportional to the transparency of the corresponding line, i.e., the higher the probability, the darker the line appears. Based on the known anatomy of the human brain, the recovered connectivity paths follow the true fiber tracts in all the experiments performed. While both the global and local algorithms provide connectivity paths that are similar, the com-

\footnotetext{
${ }^{1}$ The reader is encouraged to zoom-in on the figures in the electronic version to get a better sense of the $3 \mathrm{D}$ characteristics and the transparency values.
} 


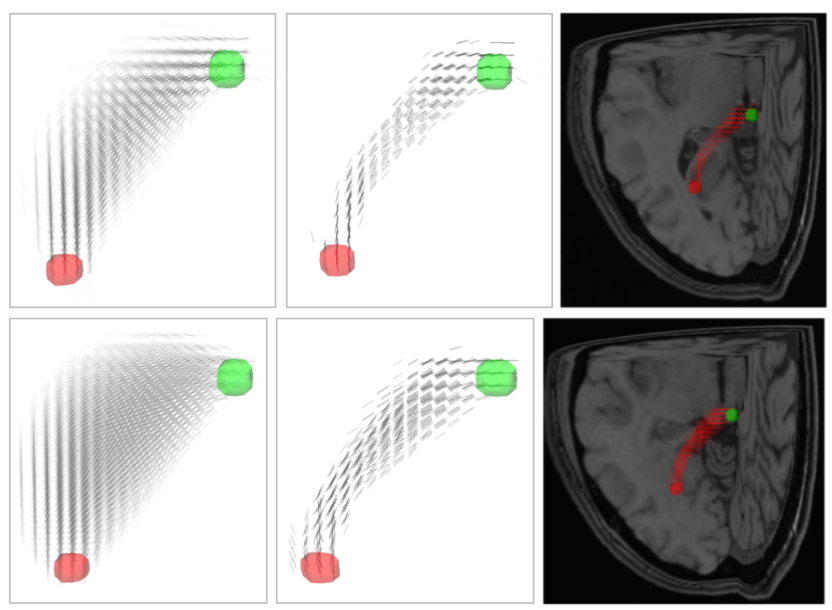

Figure 5. Inference of the connection between a pair of source and sink regions through part of the corpus callosum. Top row: Sequential algorithm. Bottom row: Local Algorithm. From left to right: (1)Before incorporating the Q-Ball data, (2)Final connectivity path after incorporating the Q-Ball data, (3)Final connectivity path overlaid on the T1-weighted image for reference.

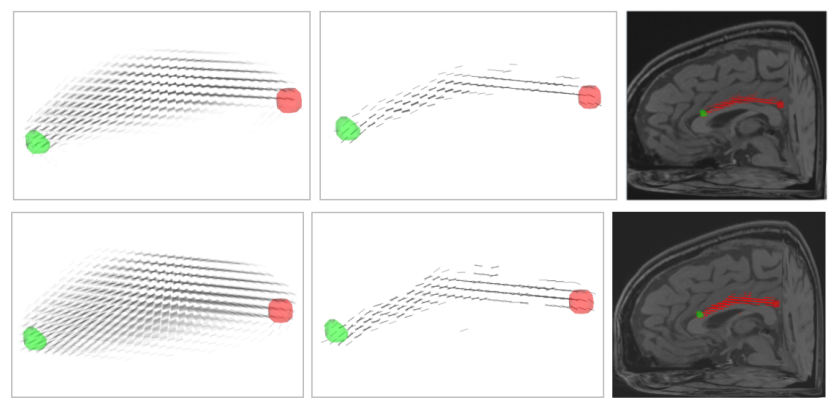

Figure 6. Inference of the connection between a pair of source and sink regions through part of the cingulum. Top row: Sequential algorithm. Bottom row: Local Algorithm. From left to right: (1)Before incorporating the Q-Ball data, (2)Final connectivity path after incorporating the Q-Ball data, (3)Final connectivity path overlaid on the T1-weighted image for reference.

pletion fields generated by the global algorithm seem to have a narrower spread in all the cases examined. Moreover, the probability values associated with the underlying states differ between the two algorithms. This observation can be explained by the fact that although the number of trials used in the Monte-Carlo simulation of the global method proved to be sufficient for the extraction of the connectivity pathways, the recovery of the true probability values may require a larger number of trials. Since the local method solves the differential equation of the underlying 3D random walk, the corresponding estimated probabilities are a better approximation of the true connectivity.

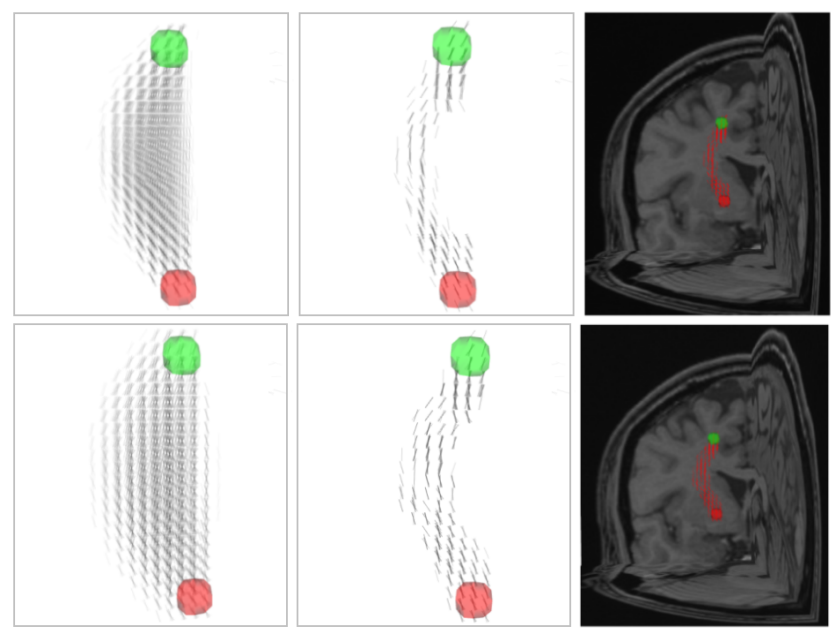

Figure 7. Inference of the connection between a pair of source and sink regions through part of the cortico-spinal tract. Top row: Sequential algorithm. Bottom row: Local Algorithm. From left to right: (1)Before incorporating the Q-Ball data, (2)Final connectivity path after incorporating the Q-Ball data, (3)Final connectivity path overlaid on the T1-weighted image for reference.

\section{Discussion}

The experiments reported in the previous section, though visually plausible, are nevertheless preliminary. Clearly, more experimental work has to be carried out to assess the utility of stochastic completion field tractography, including comparisons with other methods. Nevertheless, we are encouraged by the results obtained thus far and we believe that a framework which models the diffusion process, along with a probabilistic notion of likely fiber paths, as we have done in this paper, is the basis of a more principled approach for anatomical connectivity than has been reported in the literature thus far. Our view is supported in philosophy by some of the very recent tractography literature, including a more heuristic formulation that combines probability maps [9] and a minimal path approach [25]. Computationally there is also a connection between stochastic completion fields and the idea of tensor voting, as has been pointed out by van Almsick et al. for the 2D case [22].

Tracing more subtle connectivity pathways between different cortical-subcortical ROIs and formulating a confidence measure for the generated fiber tracts based on the recovered probability values are both subjects of ongoing work. We also intend to replace Q-Ball ODFs with fiber ODFs, which are known to better represent the true fiber structure. Our local method also allows for the incorporation of local diffusion parameters $\left(\sigma_{\theta}, \sigma_{\phi}\right)$ from the underlying data, and this might lead to more efficient and informative solutions. Currently, the parameters used throughout the experiments are chosen experimentally and are based on 
anatomical knowledge that the fibers are not highly curved for the tract systems considered. The incorporation of diffusion data and anatomical knowledge into the local method would allow for automatic selection of the required parameters.

Finally, while in this paper we have illustrated the proposed algorithms with tractography results, our future objective is to compute a reliable measure of connectivity between given source and sink regions. We expect that while variations in the size and location of the source and sink regions will effect the visualized pathways, the derived connectivity measures will be less sensitive to such changes.

Acknowledgments We are grateful to Jennifer Campbell and Bruce Pike for many helpful discussions and for providing the QBI data for our experiments. This work was supported by grants from NSERC and FQRNT Québec.

\section{References}

[1] P. Basser, S. Pajevic, C. Pierpaoli, J. Duda, and A. Aldroubi. In vitro fiber tractography using DT-MRI data. Magn. Reson. Med., 44:625-632, 2000.

[2] T. Behrens. Probabilistic diffusion tractography with multiple fibre orientations: what can we gain? NeuroImage, 34:144-155, 2007.

[3] T. Behrens, M. Woolrich, M. Jenkinson, H. Johansen-Berg, and R. N. et al. Characterization and propagation of uncertainty in diffusion-weighted MR imaging. Magn. Reson. Med., 50:1077-1088, 2003.

[4] J. Berman, S. Chung, P. Mukherjee, C. Hess, E. Han, and R. Henrya. Probabilistic streamline q-ball tractography using the residual bootstrap. NeuroImage, 39:215-222, 2008.

[5] J. Campbell, K. Siddiqi, V. Rymar, A. Sadikot, and B. Pike. Flow-based fiber tracking with diffusion tensor q-ball data: validation and comparison to principal diffusion direction techniques. NeuroImage, 27:725-736, 2005.

[6] Y. Cointepas, C. Poupon, D. L. Bihan, and J. Mangin. A spin glass framework to untangle fiber crossing in MR diffusion based tracking. In Proceedings of the MICCAI 2002, pages 475-482, Tokyo, Japan, sep 2002.

[7] R. Deriche and M. Descoteaux. Splitting tracking through crossing fibers: Multidirectional q-ball tracking. Biomedical Imaging: From Nano to Macro, 2007.

[8] D. Jones and C. Pierpaoli. Confidence mapping in diffusion tensor magnetic resonance imaging tractography using a bootstrap approach. Magn. Reson. Med., 53:1143-1149, 2005.

[9] B. W. Kreher, S. Schnell, I. Mader, K. A. Il'yasov, J. Hennig, V. G. Kiselev, and D. Saur. Connecting and merging fibres: Pathway extraction by combining probability maps. NeuroImage, 43:81-89, 2008.

[10] M. Lazar and A. Alexander. Bootstrap white matter tractography (boottrac). NeuroImage, 24:524-532, 2005.

[11] D. LeBihan, E. Breton, D. Lallemand, P. Grenier, E. Cabanis, and M. Laval-Jeantet. MR imaging of intravoxel incoherent motions: application to diffusion and perfusion in neurologic disorders. Radiology, 161:401-407, Nov. 1986.

[12] D. LeBihan, R. Turner, and P. Douek. Is water diffusion restricted in human brain white matter? an echo-planar NMR imaging study. NeuroReport, 4:887-890, July 1993.

[13] S. Mori, B. Crain, V. Chacko, and P. van Zijl. Three dimensional tracking of axonal projections in the brain by magnetic resonance imaging. Ann. Neurol., 45:265-269, 1999.

[14] D. Mumford. Algebraic Geometry and Its Applications, chapter (31):Elastica and computer vision, pages 491-506. Springer-Verlag, New York, 1994.

[15] G. Parker and D. Alexander. Probabilistic Monte Carlo based mapping of cerebral connections utilising whole-brain crossing fibre information. In Proceedings of IPMI, pages 684 695, Ambleside, UK, jul 2003.

[16] G. Parker, C. Wheeler-Kingshott, and G. Barker. Estimating distributed anatomical connectivity using fast marching methods and diffusion tensor imaging. Trans. Med. Imaging, 21:505-512, 2002.

[17] C. Poupon, C. Clark, V. Frouin, J. Regis, I. Bloch, D. LeBihan, and J. Mangin. Regularization of diffusion-based direction maps for the tracking of brain white matter fascicles. NeuroImage, 12:184-195, 2000.

[18] H. Risken and T. Frank. The Fokker-Planck Equation: Methods of Solutions and Applications, volume XIV of Series in Synergetics. Springer, 1996.

[19] P. Savadjiev, J. Campbell, and B. P. K. Siddiqi. 3d curve inference for diffusion MRI regularization and fibre tractography. Med. Image Anal., 10:799-813, Aug. 2006.

[20] J. Tournier, F. Calamante, D. Gadian, and A. Connelly. Direct estimation of the fiber orientation density function from diffusion-weighted MRI data using spherical deconvolution. NeuroImage, 23:1176-1185, Sept. 2004.

[21] D. S. Tuch. Q-ball imaging. Magn. Reson. Med., 52:13581372, Dec. 2004.

[22] M. van Almsick, R. Duits, E. Franken, and B. ter Haar Romeny. From stochastic completion fields to tensor voting. In Proceedings of DSSCV, volume LNCS, 3753, pages 124-134, Maastricht, the Netherlands, 2005.

[23] L. Williams and D. Jacobs. Local parallel computation of stochastic completion fields. Neural Computation, 9:859881, 1997.

[24] L. Williams and D. Jacobs. Stochastic completion fields: A neural model of illusory contour shape and salience. Neural Computation, 9:837-858, 1997.

[25] A. Zalesky. Dt-mri fiber tracking: A shortest paths approach. IEEE Transactions on Medical Imaging, 27(10):1458-1471, October 2008. 